\title{
Assessment of residual-limb volume change using bioimpedence
}

\author{
Joan E. Sanders, PhD; ${ }^{1 *}$ Ellen L. Rogers, MS; ${ }^{1}$ Daniel C. Abrahamson, CPO ${ }^{\mathbf{2}}$ \\ Departments of ${ }^{1}$ Bioengineering and ${ }^{2}$ Rehabilitation Medicine, University of Washington, Seattle, WA
}

\begin{abstract}
We investigated electrical bioimpedance as a potential measurement modality to assess residual-limb volume change in lower-limb amputees. Four strip electrodes were positioned across the anterior lateral to posterior lateral aspects of the proximal lower leg or residual limb such that the outer pair applied current and the inner pair sensed voltage. A commercial bioimpedance analyzer supplied current at 50 frequencies between $5 \mathrm{kHz}$ and $1 \mathrm{MHz}$ and then used a wellvalidated model to determine fluid resistance. From these data, extracellular fluid volume $\left(V_{\mathrm{ECF}}\right)$ could be estimated. Bench test evaluation showed the instrument to have a root-meansquare error of less than $0.014 \%$ over a $1 \mathrm{~h}$ interval. Tests of subjects who had been transtibial amputees for at least $2 \mathrm{yr}$ showed $V_{\text {ECF }}$ changes from postural adjustments well outside the instrument error and normal minute-to-minute biological variability. The rate of $V_{\mathrm{ECF}}$ change while standing with the prosthesis donned was greater for diabetic subjects than for nondiabetic subjects. Bioimpedance analysis may have use in prosthetics research, where comparing residual-limb volume at different time points or under different treatment conditions is of interest.
\end{abstract}

Key words: amputee, bioelectrical impedance, extracellular fluid, measurement, prosthesis, rehabilitation, residual-limb volume, shrinkage, swelling, transtibial.

\section{INTRODUCTION}

Residual-limb volume fluctuation creates challenges for lower-limb prosthesis users. Limb shrinkage can cause the socket to become loose and concentrate interface stress in soft tissues over bony prominences. Limb enlargement can induce excessive pressures and occlude blood flow, denying tissues of nutrients and restricting venous return. Both of these conditions can result in soft tissue injury. Residual-limb volume fluctuation has been recognized as a major challenge that should be a priority in prosthetics research [1].

Existing treatments for managing residual-limb volume change include stump socks, air-filled inserts (e.g., Pneu-Fit, Prosthetic Concepts, Little Rock, Arkansas; Pump-It-Up!, Love Associates Inc, Batavia, New York), fluid-filled inserts (e.g., Active Contact System, Simbex, Lebanon, New Hampshire; Volume Management Pads, Ohio Willow Wood, Mount Sterling, Ohio), and vacuumassist devices (e.g., Harmony System, Otto Bock, Minneapolis, Minnesota). Although these treatments are used clinically, improvement in their performance is still needed. Important challenges for managing residual-limb volume change include selecting the proper sock thickness or insert volume, timing the ply changes correctly, and ensuring consistent performance. The treatment also needs to be matched to the individual patient-one treatment

\footnotetext{
Abbreviations: $\mathrm{CM}=$ cell membrane, $\mathrm{DA}=$ diabetic amputee, $\mathrm{ECF}=$ extracellular fluid, $\mathrm{ICF}=$ intracellular fluid, MRI = magnetic resonance imaging, $\mathrm{N}=$ nonamputee, $\mathrm{NA}=$ nondiabetic amputee, $R_{\mathrm{ECF}}=$ ECF resistance, $R_{\mathrm{ICF}}=\mathrm{ICF}$ resistance, $R_{\mathrm{TOT}}=$ total resistance of all conducting tissue, RMS $=$ rootmean-square, $\mathrm{SD}=$ standard deviation, $V_{\mathrm{ECF}}=\mathrm{ECF}$ volume.

${ }^{*}$ Address all correspondence to Joan E. Sanders, PhD, Associate Professor; Bioengineering 355061, Foege N430J, 1705 NE Pacific St, University of Washington, Seattle, WA 98195; 206-221-5872; fax: 206-616-2509.

Email: jsanders@u.washington.edu

DOI: 10.1682/JRRD.2006.08.0096
} 
may be effective for one patient but not another. For progress to be made in treatment technologies and individual case prescription, a convenient means for accurate high-resolution assessment of residual-limb volume is needed.

Most viable methods for measuring limb volume change suffer from important limitations in their usefulness to prosthetics research and practice. Radiological and magnetic resonance imaging (MRI) methods require large, expensive, specialized equipment not typically available to most prosthetists. Further, these methods may require supine positioning that can affect the measurement of interest. Laser and optical imaging methods do not provide sufficient resolution and/or take too long [2]. Perhaps the most important limitation of these methods with regard to the application of interest here is that they require the residual limb to be removed from the socket. This is a limitation because the volume of the residual limb while it is in the socket is the parameter of primary clinical interest. Residual-limb volume changes drastically once the prosthesis is doffed [3].

This research pursues a method for assessing residuallimb volume change while the residual limb is within the prosthetic socket. Our intent was to describe the modality, assess the instrument error, and then determine if volume changes measured on nondisabled and amputee subjects were outside of the instrument error.

\section{ELECTRICAL BIOIMPEDANCE}

Bioimpedance is a noninvasive means for assessing extracellular fluid (ECF) and intracellular fluid (ICF) within living tissue. Bioimpedance analysis has been used as a tool for body composition/body fat analysis [4-9] and in the assessment of fluid imbalance in hemodialysis patients [10-13]. It has not yet been applied to the prosthetics field.

Biological conductivity occurs primarily through fatfree tissue and fluids [14]. Other elements, such as bone and adipose tissue, are essentially nonconducting. By applying a low current $(<700 \mu \mathrm{A})$ through the body and measuring the resulting voltage change, we can determine the resistance of the fat-free tissue and body fluids. ECF and ICF can be distinguished because of the capacitive nature of cell membranes (CMs). CMs are not well penetrated by current applied at low frequencies. Thus, when low or zero frequency current is applied, the measured resistance corresponds primarily to ECF resistance $\left(R_{\mathrm{ECF}}\right)$. At high frequencies, current enters both ECF and ICF spaces so that measured resistance is equal to the total resistance of all conducting tissue $\left(R_{\mathrm{TOT}}\right)$. The Cole-Cole approach is commonly used for interpretation of these data [15]. Tissue is modeled as an equivalent electric circuit consisting of $R_{\mathrm{ECF}}$, ICF resistance ( $R_{\mathrm{ICF}}$ ), and a capacitance corresponding to CMs (Figure 1(a)). $R_{\mathrm{ICF}}$ is determined from $R_{\mathrm{ECF}}$ and $R_{\mathrm{TOT}}$ by

$$
\frac{1}{R_{\mathrm{TOT}}}=\frac{1}{R_{\mathrm{ECF}}}+\frac{1}{R_{\mathrm{ICF}}} .
$$

Software supplied with the commercial bioimpedance analyzer calculates $R_{\mathrm{ECF}}$ and $R_{\mathrm{ICF}}$ based on a modified

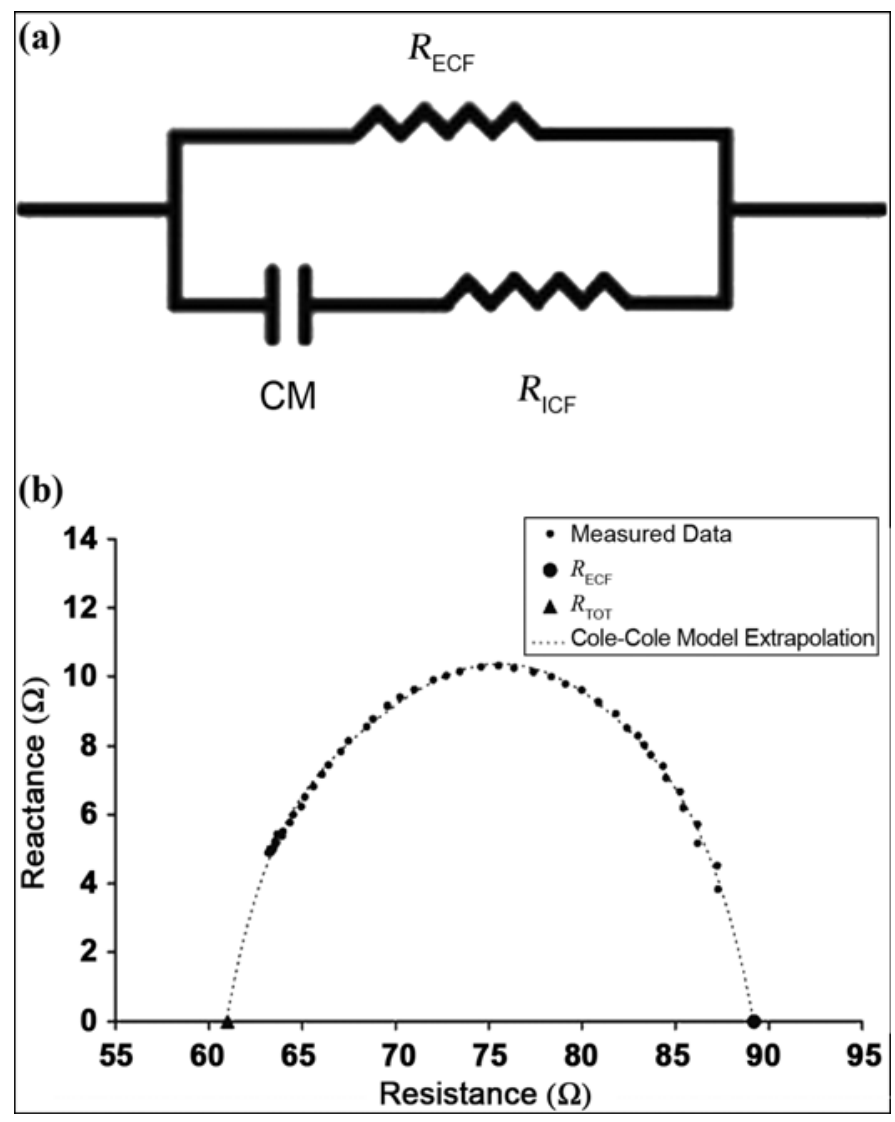

Figure 1.

(a) Schematic for Cole-Cole model. Tissue is modeled as extracellular fluid resistance $\left(R_{\mathrm{ECF}}\right)$ in parallel with intracellular fluid resistance $\left(R_{\mathrm{ICF}}\right)$ and cell membrane capacitance (CM). (b) Reactance versus resistance measured in nondisabled subject. Using Cole-Cole model, impedance data is extrapolated to high- and low-frequency limits to determine $R_{\mathrm{ECF}}$ and total resistance of all conducting tissue $\left(R_{\mathrm{TOT}}\right)$. 
Cole-Cole model. Nonlinear weighted least-squares curve-fitting applied to the multifrequency impedance spectrum is used to extrapolate $R_{\mathrm{ECF}}$ and $R_{\mathrm{TOT}}$ at the lowand high-frequency limits (Figure 1(b)) [16-17]. A similar approach described by Gilbert et al. [18] and Siconolfi et al. [19] can be used to verify the Cole-Cole calculations. With Siconolfi et al.'s method, $R_{\mathrm{ECF}}$ is determined by the intercept with the resistance axis of the third order least-squares regression of resistance and frequency. This method has been shown to produce similar results to the Cole-Cole method [18].

Resistance determined by bioimpedance analysis is inversely related to fluid volume [14]. Fluid volume is estimated through modeling based on the principle of volume conduction $[5,20]$. Assuming a limb segment is approximately cylindrical and homogeneous, we can approximate ECF volume $\left(V_{\mathrm{ECF}}\right)$ in the limb by

$$
V=\frac{\rho L^{2}}{R},
$$

where $V$ is limb fluid volume, $R$ is fluid resistance, $L$ is the limb segment length, and $\rho$ is the specific resistivity of the biofluid. A more accurate model can be used to account for the nonhomogeneity of nonconducting elements within the limb (such as bone and adipose tissue). Mixture theory is used to distinguish between the resistivity of the conducting medium and the resistivity of the nonconducting elements [16,21]. The resulting equation for segmental $V_{\mathrm{ECF}}$ is fully derived by Fenech and Jaffrin [22]

$$
V_{\mathrm{ECF}}=\left(\frac{1}{1000}\right)\left(\frac{\rho_{(\mathrm{ECF})} C}{R_{\mathrm{ECF}}}\right)^{2 / 3} \frac{L^{5 / 3}}{(4 \pi)^{1 / 3}},
$$

where the limb segment is assumed to be a cylinder with an average circumference $C$ and length $L$. $R_{\mathrm{ECF}}$ is determined by bioimpedance analysis and $\rho_{(\mathrm{ECF})}$ is the specific resistivity of the ECF of the limb. To account for the conical shape of lower-limb segments, we can further adapt Equation (3) to approximate the limb segment as a truncated cone rather than a cylinder. However, $V_{\text {ECF }}$ calculated using both shapes yielded highly similar results [22]. The cylindrical shape assumption was used here.

The primary fluids responsible for residual-limb volume change are blood and interstitial fluid [3]. Interstitial fluid is plasma that enters and leaves the interstitial space. Changes in blood and interstitial fluid levels will be reflected primarily in the $V_{\mathrm{ECF}}$ measurement; thus $V_{\mathrm{ECF}}$ is the parameter of interest in this application.
Bioimpedance analysis has been performed in various body segments, including the leg, arm, and trunk [5,2324]. Segmental bioimpedance analysis has been used successfully to determine changes in $V_{\mathrm{ECF}}$ with respect to body position [12,22-23]. $R_{\mathrm{ECF}}$ increased and $V_{\mathrm{ECF}}$ decreased in the legs when subjects changed body position from sitting to supine to supine with elevated legs, indicating that fluid was redistributed from the legs into the trunk during supine positioning and leg elevation [22]. In another study, Zhu et al. determined the $R_{\mathrm{ECF}}$ in the calf while a blood pressure cuff restricted blood flow [25]. $R_{\mathrm{ECF}}$ was found to be higher while the calf was under pressure, indicating that $V_{\mathrm{ECF}}$ was reduced by the restriction of blood flow into the region.

Bioimpedance analysis models for calculation of extracellular resistance and prediction of whole body extracellular water volume have been validated using deuterium oxide [5,26-28] and bromide dilution techniques. With bromide dilution, considered the gold standard for $V_{\mathrm{ECF}}$ assessment, whole body $V_{\mathrm{ECF}}$ is determined from bromide concentrations in blood plasma following solution ingestion $[19,28]$. Bromide dilution- and bioimpedance-based $V_{\mathrm{ECF}}$ were found to be highly correlated ( $r>$ 0.9) [19]. Validation for the calculation of $V_{\mathrm{ECF}}$ in a single limb segment has also been done. Limb-segment muscle volume predicted from bioimpedance-based calculations of $R_{\mathrm{ECF}}$ and $R_{\mathrm{ICF}}$ [6-7] was highly correlated ( $\left.r>0.9\right)$ with muscle volume determined from MRI [29].

\section{METHODS}

\section{Subjects}

Two nondisabled nonamputee $(\mathrm{N})$ subjects and four amputee subjects (two diabetic amputee [DA] and two nondiabetic amputee [NA]) participated in this research (Table 1). The nondisabled subjects were 25 and 24 years of age and had no relevant physical disability or health problems. The amputee subjects were between 58 and 64 years of age and had had their amputations for at least 2 years. The two diabetic subjects had been diagnosed for at least 6 years, although the cause of subject DA1's amputation was traumatic injury rather than diabetes. This study was approved by the University of Washington Human Subjects Division and informed consent was obtained. 
Table 1.

Subject characteristics.

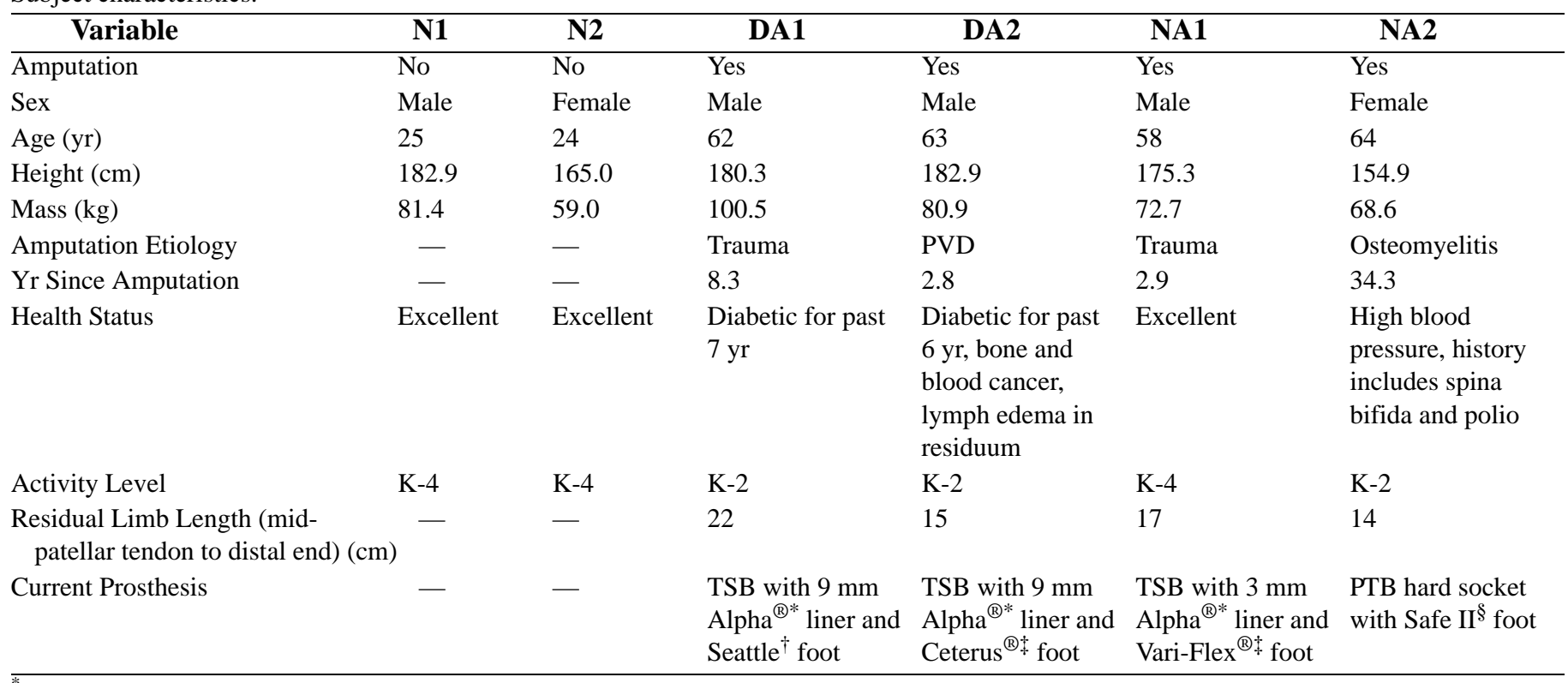

*ohio Willow Wood, Mount Sterling, Ohio.

${ }^{\dagger}$ Seattle Systems Inc, Poulsbo, Washington.

${ }_{\ddagger}$ Ossur, Reykjavik, Iceland.

${ }^{\S}$ Foresee Orthopedic Products, Oakdale, California.

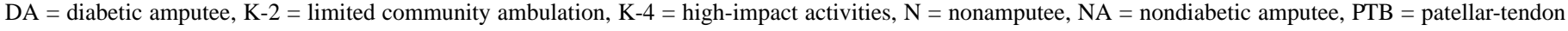
bearing, $\mathrm{PVD}=$ peripheral vascular disease, TSB = total-surface bearing.

\section{Instrumentation}

A commercial bioimpedance measurement instrument, the Xitron Hydra 4200 (Xitron Technologies, San Diego, California), was used. The Xitron Hydra 4200 is a highresolution bioimpedance analyzer that measures impedance at 50 frequencies between $5 \mathrm{kHz}$ and $1 \mathrm{MHz}$. A fourelectrode configuration was used to collect impedance data. Current was applied between two outer electrodes, while two inner electrodes sensed voltage (Figure 2).

Strip electrodes $(7.7 \mathrm{~cm} \times 2.0 \mathrm{~cm}$ contact surface, $0.081 \mathrm{~cm}$ thickness) were used in all test sessions. We used these as opposed to spot electrodes because strip electrodes distribute current over a wider range of the limb and thus provide an assessment of the limb cross section as opposed to just one region. Instead of connecting the commercial clips provided with the instrument to the electrodes, we soldered $40 \mathrm{~cm}$-long, 24-gauge multistranded wire segments to each electrode end. The Xitron Hydra 4200 leads were then clipped to the other ends of these 24-gauge wires. This modification was necessary for us to record bioimpedance while subjects were in their prosthetic sockets because the commercial clips provided with the instrument were too large. The electrode connections and wires, when positioned over fleshy sites, did not cause discomfort to the subjects when the socket was worn. Further, our modifications ensured that the wires remained attached to the electrodes at a consistent location during the different activities within a test session, which reduced error.

Prior to data collection, we allowed the instrument to warm-up for $1 \mathrm{~h}$. We acquired samples at $1 \mathrm{~Hz}$ using a laptop computer data acquisition system (D620, Latitude, 1.66 GHz; Dell Inc, Round Rock, Texas). We processed all data with the Cole-Cole model using the software provided by Xitron and then verified the data with Siconolfi et al.’s model.

\section{Bench Tests}

To quantify error inherent to the Xitron Hydra 4200 instrument, we conducted bench tests using a circuit model. The model consisted of two $8 \mathrm{~cm} \times 8 \mathrm{~cm}$ pieces of adhesive copper tape with a $100 \Omega$ carbon-film resistor soldered between their adjacent edges. The copper-tape pieces were adhered to a nonconducting wood block such 


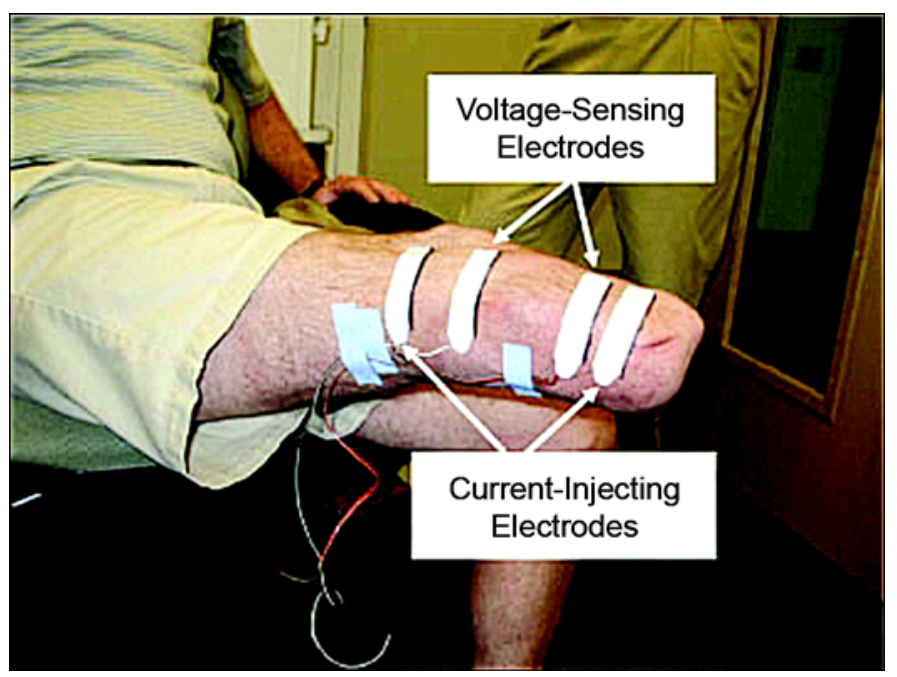

Figure 2.

Electrode setup. Two outer electrodes were current-injecting, while two inner electrodes were voltage-sensing. For testing on nondisabled subjects, similar positions on proximal lower leg were used.

that their edges were parallel to each other, with an edgeto-edge spacing of $2.5 \mathrm{~cm}$. Four strip electrodes prepared with coupling gel (Couplant D; Panametrics, NDT, Waltham, Massachusetts) on their bottom surfaces and 24-gauge multistranded wire attached to the tab on each were applied to the copper-tape pieces, two on each piece. The leads of the Xitron Hydra 4200 were clipped to the free ends of each 24-gauge wire such that the outer electrode pair applied current and the inner electrode pair sensed voltage.

To assess drift in the instrument, we collected data at $1 \mathrm{~Hz}$ over a $1 \mathrm{~h}$ interval. Variability in the data (standard deviation $[\mathrm{SD}] /$ mean) was determined. We used a linear least-squares fit to the data to quantify drift.

To assess repeatability, we collected data at $1 \mathrm{~Hz}$ for $90 \mathrm{~s}$, unclipped the leads from the wires for 60 to $360 \mathrm{~s}$, and then collected data again for $90 \mathrm{~s}$. This process was repeated until five $90 \mathrm{~s}$ data-collection intervals were completed. Mean impedance was calculated for each data collection interval and then compared.

\section{Evaluations on Nondisabled Subjects}

While the bench tests quantified error inherent in the Xitron Hydra 4200 instrument, we conducted additional tests on human subjects to evaluate the influence of protocol design on the measurements. We evaluated electrode removal and reapplication and the effects of altering the distances between the electrodes. These results were useful for the design of subsequent amputee subject test sessions.

Two nondisabled subjects participated in this aspect of the study (Table 1). The posterior lateral proximal portion of the lower leg where the electrodes were to be positioned was cleaned with an alcohol swab before electrode application. Electrodes were prepared by applying a small quantity of coupling gel to the bottom surfaces and then positioning the electrodes across the anterior lateral and posterior lateral aspects of the proximal lower leg. The coupling gel facilitated electrical contact between the skin and the electrodes. Preliminary testing without the coupling gel showed excessive noise in the data. Unless stated otherwise, all nondisabled subject tests were conducted at segment lengths (voltage electrode center-to-center spacing) of $10.0 \mathrm{~cm}$.

\section{Repeatability (Biological Variability)}

Repeatability, perhaps better termed biological variability since it is not instrumentation error, was assessed with the subject standing and bearing equal weight on each leg. After a $10 \mathrm{~min}$ period to establish homeostasis, we collected data for $5 \mathrm{~s}$. A 10 min homeostasis interval was selected because preliminary testing on nondisabled subjects under continuous data collection showed minimal change after $10 \mathrm{~min}$. After $15 \mathrm{~s}$, we collected data for another $5 \mathrm{~s}$ interval. This process was repeated until five 5 s data-collection periods were completed. The mean $R_{\mathrm{ECF}}$ and $V_{\mathrm{ECF}}$ for each $5 \mathrm{~s}$ data-collection period were calculated.

\section{Electrode Removal and Reapplication}

After a 10 min period to establish homeostasis, we performed the assessment while the subject stood with equal weight-bearing. The electrodes were positioned on the limb as described previously, and electrode positions were marked with a surgical marker. Data were collected for $5 \mathrm{~s}$, and then all four electrodes were removed and replaced with new electrodes and new coupling gel, a process that took a few minutes. Data were collected again for $5 \mathrm{~s}$. Mean $R_{\mathrm{ECF}}$ and $V_{\mathrm{ECF}}$ for each $5 \mathrm{~s}$ data collection period were calculated.

\section{Segment Length}

To test the effect of segment length, i.e., distance between the voltage-sensing electrodes, we assessed bioimpedance on the proximal lower limb using segment 
lengths of 10.0 and $5.5 \mathrm{~cm}$. Three different postures were tested: supine; sitting with leg elevated, i.e., horizontal leg supported at the heel by a chair; and standing. Postures were held for approximately 10 min prior to recording data for $5 \mathrm{~s}$.

\section{Current-Injecting and Voltage-Sensing Electrode Spacing}

The center-to-center distance between the distal voltage-sensing and current-injecting electrodes was varied between 2.0 and $5.0 \mathrm{~cm}$ in $1.0 \mathrm{~cm}$ increments, with the segment length at $10.0 \mathrm{~cm}$. For this assessment, the subject was in a seated position with the measured leg horizontal, supported by the heel on a chair. Data were collected for $5 \mathrm{~s}$ at each distance.

\section{Pressure Application}

An additional test on nondisabled subjects was conducted to evaluate impedance changes under pressure application. A blood pressure cuff (SM-500; JAC Healthcare, Vernon, California) was used to apply pressure around the proximal lower leg. During a test, the subject rested supine on a table. After a 10 min period to establish homeostasis, we applied pressure in $40 \mathrm{mmHg}$ increments over a range of 0-200 mmHg. Pressures were held for approximately $30 \mathrm{~s}$ at each level. After the $200 \mathrm{mmHg}$ pressure level was tested, the pressure was released and the cuff removed and data were collected for an additional $4.0 \mathrm{~min}$. Data were recorded continuously over this 8.0 to 8.5 min trial.

\section{Evaluations on Amputee Subjects}

Tests on the four amputee subjects (Table 1) were conducted to assess bioimpedance changes in different postures. Subjects were monitored between 9 am and $12 \mathrm{pm}$.

During a session, the subject first sat quietly in a chair with his or her normal prosthesis donned for $10 \mathrm{~min}$. The purpose of this rest interval was to establish a homeostatic condition at the beginning of testing. The subject then removed his or her prosthesis, the skin where electrodes were to be placed was cleaned with an alcohol swab, and the electrodes (with coupling gel) were placed on the residual limb. Data collection was then initiated and continued through the rest of the session. The subject donned his or her liner and prosthesis. He or she then stood with equal weight-bearing for $5 \mathrm{~min}$, followed by in-place walking for $5 \mathrm{~min}$. In-place walking was one step forward with the prosthetic limb and then one step backward. The subject lifted the prosthetic limb off of the ground after taking the one step forward in each cycle. The reason inplace walking as opposed to ambulation was evaluated was that the subject was tethered to the data acquisition system via the instrument leads. In future testing, a treadmill or wireless data transmission system could be used to overcome this limitation and allow for ambulation. The subject then sat down and immediately removed his or her socket and liner. The subject then sat quietly with the residual limb dependent for $5 \mathrm{~min}$. The data collection part of the session lasted a total of 15 to $20 \mathrm{~min}$. We processed the data to determine a $V_{\mathrm{ECF}}$ versus time curve for the entire session. $V_{\mathrm{ECF}}$ values at the following time points were determined: (1) while sitting, immediately prior to donning the prosthesis; (2) while standing, $2.5 \mathrm{~min}$ after standing up; (3) while walking, just before sitting down; and (4) while resting with the prosthesis off and residual limb dependent, $2.5 \mathrm{~min}$ after doffing the prosthesis. The rate of change of $V_{\mathrm{ECF}}, V_{\mathrm{ECF}} /$ time, was determined during standing (2.0 min after point 2) and during resting (2.0 min after point 4).

\section{RESULTS}

\section{Bench Tests}

Bench test results from the circuit model showed that variability in the $R_{\mathrm{ECF}}$ measurement (SD/mean) over a $1 \mathrm{~h}$ interval was 0.005 percent. Drift was $0.012 \Omega / \mathrm{h}$ $(0.012 \% / h)$. Variability in the five repeatability tests (five $90 \mathrm{~s}$ data-collection intervals spaced at least $60 \mathrm{~s}$ apart) from the circuit model was 0.011 percent. Thus, root-mean-square (RMS) error assessed over a $1 \mathrm{~h}$ interval was $<0.014$ percent.

\section{Evaluations on Nondisabled Subjects}

Evaluation on nondisabled subjects showed that variability in $R_{\mathrm{ECF}}$ for the five repeated tests (subjects standing) was 0.2 percent for subject $\mathrm{N} 1$ and 0.3 percent for subject N2. Using the Fenech and Jaffrin model for segmental volume [22], we calculated mean $V_{\mathrm{ECF}}$ as $238.5 \mathrm{~mL}$ and $207.1 \mathrm{~mL}$, respectively, for subjects $\mathrm{N} 1$ and $\mathrm{N} 2$. Thus, variability in volume units was $0.5 \mathrm{~mL}$ and $0.6 \mathrm{~mL}$, respectively.

Electrode removal and reapplication changed the $R_{\mathrm{ECF}}$ measurement by $0.05 \Omega$ for subject $\mathrm{N} 1$ and $0.12 \Omega$ for subject N2. These changes corresponded to volume differences of $0.2 \mathrm{~mL}(0.1 \%)$ and $0.6 \mathrm{~mL}(0.3 \%)$, respectively. 
$R_{\mathrm{ECF}}$ measurements were lower for the shorter segment length, as expected. For data collected for supine, sitting, and standing positions, paired differences between 10.0 and $5.5 \mathrm{~cm}$ segment lengths averaged 47.7 percent for subject $\mathrm{N} 1$ and 36.6 percent for subject N2. We should note that the Cole-Cole model and Siconolfi et al.'s model still performed well for the 5.5 cm-segment length, which validates that this segment length was acceptable. Amputees with short residual limbs may require this short electrode spacing.

$V_{\mathrm{ECF}}$ decreased 5.2 and 6.5 percent for supine versus standing and 6.1 and 8.9 percent for sitting versus standing postures for subjects N1 and N2, respectively (Table 2). These differences corresponded to limb volume changes between -11.8 and $-16.9 \mathrm{~mL}$.

The distance from the distal current-injecting electrode to the distal voltage-sensing electrode affected the measured $R_{\mathrm{ECF}}$. At a distance of $2.0 \mathrm{~cm}$, the data did not fit the Cole-Cole model well. At a distance of 3.0 to $5.0 \mathrm{~cm}$, however, the data conformed well to the model. Thus, at least $3.0 \mathrm{~cm}$ spacing was used in all test sessions. For the 3.0 to $5.0 \mathrm{~cm}$ spacing, $R_{\mathrm{ECF}}$ decreased linearly with distance by $2.7 \Omega / \mathrm{cm}$ and $2.4 \Omega / \mathrm{cm}$ for subjects $\mathrm{N} 1$ and $\mathrm{N} 2$, respectively.

Pressure application with a blood pressure cuff caused a decrease in $V_{\mathrm{ECF}}$ each time the cuff pressure was increased, which created a steplike $V_{\mathrm{ECF}}$ versus time curve (Figure 3). Over a 4.0 min interval, after the cuff was removed, $V_{\text {ECF }}$ decreased 1.3 and 2.6 percent for subjects N1 and N2, respectively.

Plotting the data as $V_{\mathrm{ECF}}$ versus pressure resulted in concave curves (Figure 4), with the rate of volume change per unit pressure decreasing with increased pressure.

Table 2.

Nonamputee (N) subject results.

\begin{tabular}{lcc}
\hline \multicolumn{1}{c}{ Posture } & N1 & N2 \\
\hline$V_{\text {ECF, }}$ mL & 226.8 & 194.4 \\
Supine & 224.9 & 190.2 \\
Sitting & \\
Standing & 238.5 & 207.1 \\
$\Delta V_{\text {ECF, }}$ mL (\%) & & $-12.7(-6.5)$ \\
Supine-to-Stand & $-11.8(-5.2)$ & $-16.9(-8.9)$ \\
$\quad$ Sit-to-Stand & $-13.7(-6.1)$ & \\
${ }^{*}$ Leg elevated. & \\
$V_{\text {ECF }}=$ extracellular fluid volume. & \\
\hline \hline
\end{tabular}

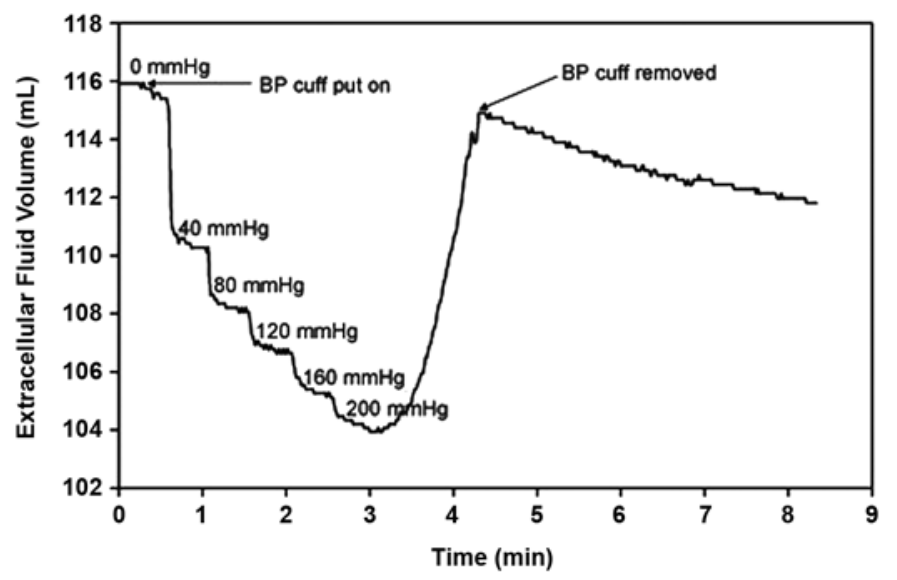

Figure 3.

Pressure application on nondisabled subject. Blood pressure (BP) cuff was applied and inflated at $40 \mathrm{mmHg}$ increments. At $200 \mathrm{mmHg}$, pressure was released, cuff removed, and data collected for additional $4 \mathrm{~min}$.

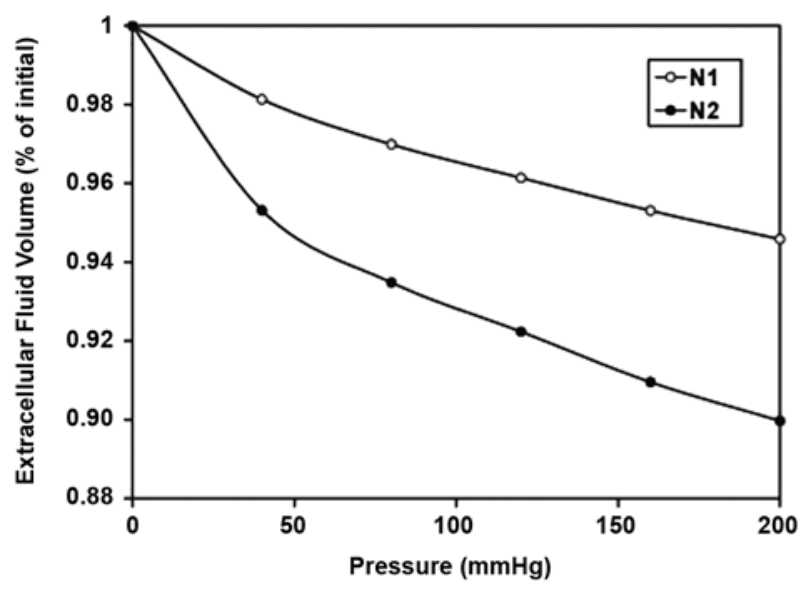

Figure 4.

Extracellular fluid volume ( $\left.V_{\mathrm{ECF}}\right)$ versus pressure for nonamputee $(\mathrm{N})$ subjects. Results from blood pressure cuff tests show nonlinear curve, with change in $V_{\mathrm{ECF}}$ decreasing as pressure increased.

\section{Evaluations on Amputee Subjects}

Amputee subject tests showed $V_{\mathrm{ECF}}$ changes over the course of the 15-to-20 min session. Results shown in Figure 5 for subject DA1 are typical. $V_{\text {ECF }}$ decreased from sit-to-stand, which was consistent with standing causing the socket to apply pressure to the residual limb and displace fluid. $V_{\mathrm{ECF}}$ then slowly decreased while the subject stood still with equal weight-bearing for several minutes. During walking, $V_{\text {ECF }}$ cycled as expected, though on occasion the signal was lost because of excessive strain to the electrode connection (Figure 5). After 


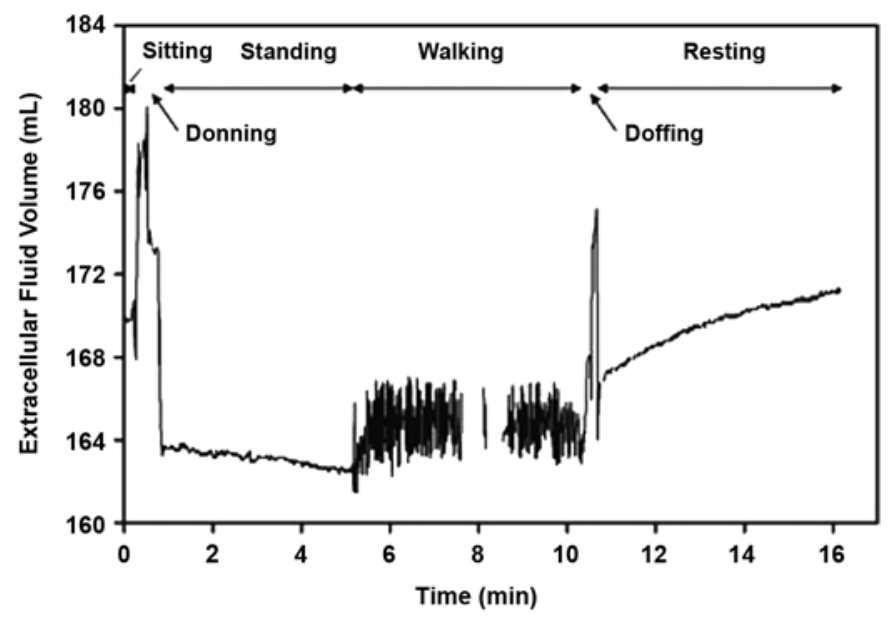

Figure 5.

Extracellular fluid volume recorded on amputee subject. After sitting, subject donned prosthesis, stood with equal weight-bearing, walked, doffed prosthesis, and rested with limb dependent.

the subject sat, doffed the prosthesis and liner, and rested with the residual limb dependent, $V_{\mathrm{ECF}}$ increased as the residual limb swelled. At $0.5 \mathrm{~min}$ and $10.5 \mathrm{~min}$, while the prosthesis and liner were donned and doffed, respectively, the data showed great fluctuation.

Results showed larger percent $V_{\mathrm{ECF}}$ changes from sit-to-stand for diabetic subjects than for nondiabetic subjects (Table 3). Diabetic subject changes were -5.9 and -6.9 percent, while nondiabetic subject changes were -3.1 and -3.6 percent. Subject DA2, who had pitted edema in his residual limb, showed very large $V_{\mathrm{ECF}}$ changes between walking and removing the prosthesis and resting (8.5\%) compared with the nondiabetic subjects $(4.0 \%$ and $0.7 \%)$. Volume differences from the beginning to the end of the study were larger for the diabetic subjects $(-2.2 \%$ and $-4.3 \%)$ than for the nondiabetic subjects $(0.4 \%$ and $-0.9 \%)$.

\section{DISCUSSION}

Bioimpedance measurement has potential value in prosthetics research and clinical practice as an effective means of measuring volume changes while the residual limb is within the prosthetic socket. Bioimpedance could potentially be used to evaluate different volume control strategies, different suspension systems, or other treatments of interest. Residual-limb volume within the socket as opposed to outside of the socket is the parameter of interest. Limb shape typically changes rapidly after the socket and liner are removed [3]; thus techniques that measure limb volume after doffing are not as useful [2].

We should note that bioimpedance measurement offers assessment of the change in limb volume as opposed to absolute limb volume. This relative measurement is acceptable, since tests of different prosthesis designs or assessments between two points in time are of greater clinical interest. Error in the Xitron Hydra 4200 instrument was minimal for this relative measurement. Our tests showed that repeatability and drift produced an RMS error of $<0.014$ percent over a $1 \mathrm{~h}$ interval. This

Table 3.

Diabetic amputee (DA) and nondiabetic amputee (NA) subject results.

\begin{tabular}{|c|c|c|c|c|}
\hline Posture & DA1 & DA2 & NA1 & NA2 \\
\hline \multicolumn{5}{|l|}{$\overline{V_{\mathrm{ECF}}}, \mathrm{mL}$} \\
\hline Sitting (1) & 173.3 & 97.2 & 111.6 & 75.5 \\
\hline Walking (3) & 163.9 & 85.7 & 107.7 & 74.3 \\
\hline Resting (4) & 169.5 & 93.0 & 112.0 & 74.8 \\
\hline \multicolumn{5}{|l|}{$\Delta V_{\mathrm{ECF}}, \mathrm{mL}(\%)$} \\
\hline Walk-to-Rest (4-3) & $5.6(3.4)$ & $7.3(8.5)$ & $4.3(4.0)$ & $0.5(0.7)$ \\
\hline Start-to-Finish (4-1) & $-3.8(-2.2)$ & $-4.2(-4.3)$ & $0.4(0.4)$ & $-0.7(-0.9)$ \\
\hline \multicolumn{5}{|c|}{$\Delta V_{\mathrm{ECF}} / t, \mathrm{~mL} / \mathrm{min}(\% / \mathrm{min})$} \\
\hline Standing (S1) & $-0.36(-0.22)$ & $-0.32(-0.36)$ & $-0.12(-0.11)$ & $-0.10(-0.14)$ \\
\hline Resting (S2) & $0.59(0.35)$ & $0.56(0.60)$ & $0.21(0.19)$ & $0.28(0.37)$ \\
\hline
\end{tabular}


value is far less than $V_{\mathrm{ECF}}$ changes induced by postural changes for amputee and nondisabled subjects (measured here between $0.7 \%$ and $8.9 \%$ ). Thus, the bioimpedance measurement described in this article is well matched to assessment of residual-limb volume change.

Biological variability, i.e., minute-to-minute change in volume as measured by bioimpedance, was outside the instrument error and thus measurable. Variability of nondisabled subjects of 0.2 or 0.3 percent for five repeated tests conducted over a 2 min interval was on the low end of the biological variability measured from all subjects in this study. Amputee subjects' residual limbs typically change size over time, particularly after the prosthesis is donned or doffed (Figure 5); the variability in their measurements was larger than that of nondisabled subjects. For amputee subjects, $V_{\text {ECF }}$ changed from 0.2 to 1.2 percent over 2 min intervals of stationary standing or resting. This result points to the need for a consistent protocol when bioimpedance testing is conducted, e.g., when different treatments are evaluated. Investigators need to take steps to minimize the influence of biological or minute-to-minute variability on the analysis of interest.

Our tests with this instrument led us to a low-end spacing threshold of $5.5 \mathrm{~cm}$ between voltage-sensing electrodes and $3.0 \mathrm{~cm}$ between current-injecting and voltagesensing electrodes. These distances make bioimpedance measurement viable for most amputee subject residuallimb lengths. For shorter distances, current was not well distributed through the segment and thus distortion and poor fits to the Cole-Cole and Siconolfi et al. models occurred. Our values are below those suggested in the Xitron Hydra 4200 instrument manual $(10.0$ and $5.0 \mathrm{~cm}$, respectively). However, because changes in bioimpedance, as opposed to absolute measurements, are of interest here and because our data fit the models, our parameter thresholds are considered acceptable for this application. We should note, however, that segment length should be made as long as possible to enhance the likelihood of well-distributed current. A long segment length also enhances the likelihood that the entire limb cross-section is assessed. Further, our data demonstrate that positioning the current-injecting electrodes a consistent distance from the voltage-sensing electrodes is crucial if comparisons are to be made between sessions.

Sensitivity to electrode removal and reapplication $(0.1 \%$ and $0.3 \%)$ was comparable to the variability measured on nondisabled subjects. Thus, exchanging an electrode because of failure or problems does not substantially affect the data as long as the replacement position is consistent. Error may result from the time taken to do so, however, because of the residual-limb swelling that may occur. A template to facilitate consistent electrode placement would potentially facilitate consistent positioning and reduce the time for electrode replacement. In addition, relieving the strain between the 24-gauge wire and the electrode tab so that the signal is not detrimentally affected during ambulation could reduce error.

Results from the blood pressure cuff tests on nondisabled subjects are consistent with findings reported in the literature [25]. $V_{\mathrm{ECF}}$ decreased as cuff pressure was increased, primarily because of reduction in blood flow under pressure. Volume change was less at very high pressures than at low pressures. The drift during constant pressure application was likely because of the viscoelastic nature of the soft tissues and the slow movement of interstitial fluid out of the limb. After the pressure cuff was removed, fluid rushed back into the limb, which brought total limb volume back to near the initial level. We suspect that a net interstitial fluid reduction over the course of pressure application was present that was not recovered immediately upon cuff removal. The slow decrease in volume after cuff removal may be a reduction in blood volume after the overcompensation of blood flow upon cuff removal. However, this interpretation is conjecture and would need to be verified with further studies. An exciting possibility is that blood transport could be distinguished from interstitial fluid transport through a combination of bioimpedance measurement and an appropriate pressure application protocol. Distinction between blood and interstitial fluid transport would further our understanding of residual limb-volume fluctuations and may allow development of treatments specific to the type of fluid gained and lost, both diurnally and in the long term.

While results concerning diabetic versus nondiabetic subjects cannot be generalized because of the small sample sizes, we should note that the differences seen here are consistent with clinical expectation. In general, the two DA subjects' fluid changes and rates of change in volume were larger than those of the two NA subjects. Diabetics often have compromised peripheral vasculature and much venous pooling compared with nondiabetics. Thus, the activity in the 15-to-20 min session caused fluid displacement out of the residual limb that was not immediately recovered, which resulted in an overall loss of fluid from the beginning to the end of the session. 
$V_{\mathrm{ECF}}$ changes were -2.2 and -4.3 percent for subject DA1 and DA2, respectively. The $V_{\mathrm{ECF}}$ difference from start to finish of the session on the healthiest amputee subject (NA1) of 0.4 percent was close to the variability measured on nondisabled subjects $(0.2 \%$ and $0.3 \%)$.

One possible limitation of bioimpedance measurement is the effect of scar tissue on the residual limb; scar tissue could impede local current flow and thus increase impedance. However, since differences in limb volume under different treatment conditions are the primary clinical interest here and scar tissue would likely have a consistent effect, the effect on the results would presumably be minimal.

Because a limited sample size was studied here, we cannot make quantitative conclusions about differences between study populations. However, the findings from this investigation indicate the potential utility of bioimpedance assessment in prosthetics research and clinical practice. Assessment should be possible on larger sample populations, and new studies, such as assessment of residual-limb volume control strategies or evaluation of different suspension techniques, could be performed. These areas await further investigation.

\section{CONCLUSIONS}

Bioimpedance analysis is a potentially useful tool for assessing residual-limb volume changes at different time points or under different treatment conditions. Results from bench tests showed the Xitron Hydra 4200 bioimpedance analyzer to have a RMS error $<0.014$ percent over a $1 \mathrm{~h}$ interval, far less than the biological variability measured on nondisabled subjects $(0.2 \%$ and $0.3 \%)$. $V_{\text {ECF }}$ changes in nondisabled subjects for supine versus standing (mean of $-5.9 \%$ ) and sitting versus standing (mean of $-7.5 \%$ ) postures were far greater than the instrument error or biological variability. Amputee subject tests showed $V_{\mathrm{ECF}}$ changes from postural shifts to be far greater than the instrument error or biological variability, which substantiates the validity of bioimpedance for amputee subject $V_{\mathrm{ECF}}$ assessment. $V_{\mathrm{ECF}}$ changes from sit-to-stand ranged from -3.1 to -6.9 percent and from walk-to-rest from 0.7 to 8.5 percent. The two DA subjects showed greater $V_{\mathrm{ECF}}$ changes than the two NA subjects during sit-to-stand and a 15-to-20 min session of standing, walking, and resting with the residual limb dependent, which was consistent with our expectations.
Fluid transport rates for DA subjects were, in general, faster than those for NA subjects. Bioimpedance assessment is a potentially useful tool in prosthetics research and clinical practice.

\section{ACKNOWLEDGMENTS}

We gratefully acknowledge advice from Dr. Albert Lozano.

This material was based on work supported by the National Institutes of Health (grant R01EB-004329).

The authors have declared that no competing interests exist.

\section{REFERENCES}

1. Michael JW, Bowker JH. Prosthetics/orthotics research for the twenty-first century: Summary of 1992 conference proceedings. J Prosthet Orthot. 1994;6:100-107.

2. Zheng YP, Mak AF, Leung AK. State-of-the-art methods for geometric and biomechanical assessment of residual limbs: A review. J Rehabil Res Dev. 2001;38(5):487-504. [PMID: 11732827]

3. Zachariah SG, Saxena R, Fergason JR, Sanders JE. Shape and volume change in the transtibial residuum over the short term: Preliminary investigation of six subjects. J Rehabil Res Dev. 2004;41(5):683-94. [PMID: 15558398]

4. Van Loan MD, Withers P, Matthie J, Mayclin PL. Use of bioimpedance spectroscopy to determine extracellular fluid, intracellular fluid, total body water, and fat-free mass. Basic Life Sci. 1993;60:67-70. [PMID: 8110166]

5. Organ LW, Bradham GB, Gore DT, Lozier SL. Segmental bioelectrical impedance analysis: Theory and application of a new technique. J Appl Physiol. 1994;77(1):98-112. [PMID: 7961281]

6. Fuller NJ, Hardingham CR, Graves M, Screaton N, Dixon AK, Ward LC, Elia M. Predicting composition of leg sections with anthropometry and bioelectrical impedance analysis, using magnetic resonance imaging as reference. Clin Sci (Lond). 1999;96(6):647-57. [PMID: 10334971]

7. Salinari S, Bertuzzi A, Mingrone G, Capristo E, Scarfone A, Greco AV, Heymsfield SB. Bioimpedance analysis: A useful technique for assessing appendicular lean soft tissue mass and distribution. J Appl Physiol. 2003;94(4):1552-56. [PMID: 12626475]

8. Dittmar M. Reliability and variability of bioimpedance measures in normal adults: Effects of age, gender, and body mass. Am J Phys Anthropol. 2003;122(4):361-70. [PMID: 14614757] 
9. Andreoli A, Melchiorri G, De Lorenzo A, Caruso I, Sinibaldi Salimei P, Guerrisi M. Bioelectrical impedance measures in different position and vs dual-energy X-ray absorptiometry (DXA). J Sports Med Phys Fitness. 2002; 42(2):186-89. [PMID: 12032414]

10. De Vries PM, Meijer JH, Vlaanderen K, Visser V, Oe PL, Donker AJ, Schneider H. Measurement of transcellular fluid shifts during haemodialysis. Part 2. In vitro and clinical evaluation. Med Biol Eng Comput. 1989;27(2):152-58. [PMID: 2601434]

11. Zhu F, Schneditz D, Wang E, Martin K, Morris AT, Levin NW. Validation of changes in extracellular volume measured during hemodialysis using a segmental bioimpedance technique. ASAIO J. 1998;44(5):M541-45. [PMID: 9804490]

12. Shulman T, Heidenheim AP, Kianfar C, Shulman SM, Lindsay RM. Preserving central blood volume: Changes in body fluid composition during hemodialysis. ASAIO J. 2001; 47(6):615-18. [PMID: 11730198$]$

13. Donadio C, Consani C, Ardini M, Bernabini G, Caprio F, Grassi G, Lucchesi A, Nerucci B. Estimate of body water compartments and of body composition in maintenance hemodialysis patients: Comparison of single and multifrequency bioimpedance analysis. J Ren Nutr. 2005;15(3): 332-44. [PMID: 16007563]

14. Nyboer J. Workable volume and flow concepts of biosegments by electrical impedance plethysmography. TIT J Life Sci. 1972;2(1):1-13. [PMID: 4680960]

15. Cole KS, Li CL, Bak AF. Electrical analogues for tissues. Exp Neurol. 1969;24(3):459-73. [PMID: 5800962]

16. De Lorenzo A, Andreoli A, Matthie J, Withers P. Predicting body cell mass with bioimpedance by using theoretical methods: A technological review. J Appl Physiol. 1997; 82(5):1542-58. [PMID: 9134904]

17. Matthie J, Zarowitz B, De Lorenzo A, Andreoli A, Katzarski K, Pan G, Withers P. Analytic assessment of the various bioimpedance methods used to estimate body water. J Appl Physiol. 1998;84(5):1801-16. [PMID: 9572833]

18. Gilbert JH, Suire SS, Gretebeck R, Wong WW, Siconolfi SF. Effect of frequency, circuit analysis and instrument on extracellular and total body resistance. Med Sci Sports Exerc. 1995;27(Suppl):S118.

19. Siconolfi SF, Gretebeck RJ, Wong WW, Pietrzyk RA, Suire SS. Assessing total body and extracellular water from bio- electrical response spectroscopy. J Appl Physiol. 1997; 82(2):704-10. [PMID: 9049756]

20. Hoffer EC, Meador CK, Simpson DC. Correlation of whole-body impedance with total body water volume. J Appl Physiol. 1969;27(4):531-34. [PMID: 4898406]

21. Hanai T. Electrical properties of emulsions. In: Sherman P, editor. Emulsion science. London (England): Academic Press; 1968. p. 354-477.

22. Fenech M, Jaffrin MY. Extracellular and intracellular volume variations during postural change measured by segmental and wrist-ankle bioimpedance spectroscopy. IEEE Trans Biomed Eng. 2004;51(1):166-75. [PMID: 14723506]

23. Zhu F, Schneditz D, Wang E, Levin NW. Dynamics of segmental extracellular volumes during changes in body position by bioimpedance analysis. J Appl Physiol. 1998;85(2): 497-504. [PMID: 9688726$]$

24. Thomas BJ, Cornish BH, Ward LC, Patterson MA. A comparison of segmental and wrist-to-ankle methodologies of bioimpedance analysis. Appl Radiat Isot. 1998;49(5-6): 477-78. [PMID: 9569519]

25. Zhu F, Sarkar S, Kaitwatcharachai C, Greenwood R, Ronco C, Levin NW. Methods and reproducibility of measurement of resistivity in the calf using regional bioimpedance analysis. Blood Purif. 2003;21(1):131-36. [PMID: 12596759]

26. Segal KR, Burastero S, Chun A, Coronel P, Pierson RN, Wang J. Estimation of extracellular and total body water by multiple-frequency bioelectrical-impedance measurement. Am J Clin Nutr. 1991;54(1):26-29. [PMID: 2058583$]$

27. Wotton MJ, Thomas BJ, Cornish BH, Ward LC. Comparison of whole body and segmental bioimpedance methodologies for estimating total body water. Ann N Y Acad Sci. 2000; 904:181-86. [PMID: 10865733]

28. Armstrong LE, Kenefick RW, Castellani JW, Riebe D, Kavouras SA, Kuznicki JT, Maresh CM. Bioimpedance spectroscopy technique: Intra-, extracellular, and total body water. Med Sci Sports Exerc. 1997;29(12):1657-63. [PMID: 9432101]

29. Miyatani M, Kanehisa H, Masuo Y, Ito M, Fukunaga T. Validity of estimating limb muscle volume by bioelectrical impedance. J Appl Physiol. 2001;91(1):386-94. [PMID: 11408456]

Submitted for publication August 17, 2006. Accepted in revised form February 20, 2007. 